\title{
THE MASLOV INDEX AS A QUADRATIC SPACE
}

\author{
Teruji Thomas
}

\begin{abstract}
Kashiwara defined the Maslov index (associated to a collection of Lagrangian subspaces of a symplectic vector space over a field $F$ ) as a class in the Witt group $W(F)$ of quadratic forms. We construct a canonical quadratic vector space in this class and show how to understand the basic properties of the Maslov index without passing to $W(F)$ - that is, more or less, how to upgrade Kashiwara's equalities in $W(F)$ to canonical isomorphisms between quadratic spaces. The quadratic space is defined using elementary linear algebra. On the other hand, it has a nice interpretation in terms of sheaf cohomology, due to A. Beilinson.
\end{abstract}

\section{Introduction.}

Let $F$ be a field of characteristic not 2. Let $W(F)$ denote the Witt group of quadratic spaces over $F$ - we will use the term quadratic space to mean a finite dimensional $F$-vector space with a non-degenerate symmetric bilinear form.

1.1. Suppose given a vector space $V$ over $F$ with a symplectic form $B$ and some Lagrangian subspaces $l_{1}, \ldots, l_{n}$ of $V$, indexed by $\mathbb{Z} / n \mathbb{Z}$.

To this data Kashiwara associated a class $\tau\left(l_{1}, \ldots, l_{n}\right) \in W(F)$ called the Maslov index of the Lagrangians (see the appendices in $[\mathrm{LV}]$ and $[\mathrm{KS}]$, and section 7 of this article). In this article we answer the following questions:

(i) How can one represent $\tau\left(l_{1}, \ldots, l_{n}\right)$ as the class of a canonically defined quadratic space?

(ii) How can one upgrade the basic equalities satisfied by $\tau$ to canonical isomorphisms between quadratic spaces?

By 'basic equalities' we mean dihedral symmetry, i.e.

$$
\tau\left(l_{1}, l_{2}, \ldots, l_{n}\right)=\tau\left(l_{2}, l_{3}, \ldots, l_{n}, l_{1}\right)=-\tau\left(l_{n}, l_{n-1}, \ldots, l_{1}\right)
$$

and the chain condition, i.e.

$$
\tau\left(l_{1}, l_{2}, \ldots, l_{n}\right)=\tau\left(l_{1}, l_{2}, \ldots, l_{k}\right)+\tau\left(l_{1}, l_{k}, \ldots, l_{n}\right)
$$

for any $k \in\{3, \ldots, n-1\}$. We also mean that when $F$ is a local field (e.g. $F=\mathbb{R}$ ),

$$
\tau\left(l_{1}, \ldots, l_{n}\right) \text { is locally constant in } l_{1}, \ldots, l_{n}
$$

$$
\text { if the dimension of } l_{i} \cap l_{i+1} \text { is fixed for each } i \in \mathbb{Z} / n \mathbb{Z} \text {. }
$$

Received by the editors May 19, 2006.

Partially supported by the University of Chicago's VIGRE grant, DMS-9977134, and by NSF grant DMS-0401164. 
1.2. In section 2 we answer question (i), constructing a quadratic space denoted $T_{1,2, \ldots, n}$ (with bilinear form $\left.q_{1,2, \ldots, n}\right)$. We re-define the Maslov index $\tau\left(l_{1}, l_{2}, \ldots, l_{n}\right.$ ) to be the class of $T_{1,2, \ldots, n}$ in $W(F)$, and in section 7 we verify that our Maslov index is the same as Kashiwara's.

We will give a concrete description of the quadratic space in section 2.2, but abstractly we may say that the vector space $T_{1,2, \ldots, n}$ is the cohomology $H^{0}(C)$ of a certain complex $C$ (13); the form $q_{1,2, \ldots, n}$ arises from the quasi-isomorphism of $C$ and its dual $C^{*}$. This point of view is explained in section 3 , where we also give a formula for the dual form on $T_{1,2, \ldots, n}^{*}$.

A sheaf-theoretic construction of $\left(T_{1,2, \ldots, n}, q_{1,2, \ldots, n}\right)$ is described in 1.4. The reader chiefly interested in this interpretation can proceed directly to 1.4 and then section 8 .

Remark. Having written this paper, we noticed that another answer to question (i) is proposed in [CLM], section 12, using topological methods when $F=\mathbb{R}$. However, one can find a counter-example to their formula for $n=5, \operatorname{dim} V=2$. Their method rather leads to our formula (10).

1.3. In sections $4,5,6$ we answer question (ii).

1.3.1. The dihedral symmetry (1) will be realized by canonical identifications

$$
\left(T_{1,2, \ldots, n}, q_{1,2, \ldots, n}\right)=\left(T_{2,3, \ldots, n, 1}, q_{2,3, \ldots, n, 1}\right)=\left(T_{n, n-1, \ldots, 1},-q_{n, n-1, \ldots, 1}\right)
$$

as described in section 4 .

1.3.2. For the chain condition (2), suppose first that $l_{1} \cap l_{k}=0$. In section 5 we describe a canonical isometric isomorphism

$$
T_{1,2, \ldots, k} \oplus T_{1, k, \ldots, n} \stackrel{\cong}{\longrightarrow} T_{1,2, \ldots, n} .
$$

To treat the case $l_{1} \cap l_{k} \neq 0$, we use the notion of quadratic subquotient: if $T$ is a quadratic space and $I$ an isotropic subspace, then $I^{\perp} / I$ is again a quadratic space, called 'the quadratic subquotient of $T$ by $I$.'

Without assuming $l_{1} \cap l_{k}=0$, we identify $T_{1,2, \ldots, k} \oplus T_{1, k, \ldots, n}$ with a quadratic subquotient of $T_{1,2, \ldots, n}$, so the following well known lemma shows that (2) holds without any assumptions.

Lemma 1. If $S$ is a quadratic subquotient of $T$ then $S$ and $T$ have the same class in $W(F)$.

Proof. Suppose that $S=I^{\perp} / I$. Choose a linear complement $M$ to $I^{\perp}$ in $T$. Then $M+I$ (a direct sum) is a hyperbolic summand of $S$, and $(M+I)^{\perp} \subset I^{\perp}$ maps isometrically onto $I^{\perp} / I$.

1.3.3. As for (3), it is not true in general that the isomorphism class of $T_{1,2, \ldots, n}$ is locally constant under the condition described. It would be enough to show that $\operatorname{dim} T_{1,2, \ldots, n}$ is locally constant (see Lemma 9), but in fact (see (14)), $\operatorname{dim} T_{1,2, \ldots, n}$ depends not only on the dimensions of $l_{i} \cap l_{i+1}$ but also on $\operatorname{dim}\left(l_{1} \cap \cdots \cap l_{n}\right)$.

In section 6 we represent $T_{1,2, \ldots, n}$ as a quadratic subquotient of another quadratic space $\tilde{T}_{1,2, \ldots, n}$ whose dimension depends only on the dimensions $\operatorname{dim} l_{i} \cap l_{i+1}$. Property (3) then follows from Lemma 1 above. 
Remark. As already mentioned in 1.2, there is a quasi-isomorphism $\Phi: C \rightarrow C^{*}$. To define $\tilde{T}_{1,2, \ldots, n}$ we factor $\Phi$ as

$$
C \stackrel{\alpha}{\longrightarrow} D \stackrel{\tilde{\Phi}}{\longrightarrow} D^{*} \stackrel{\alpha^{*}}{\longrightarrow} C^{*}
$$

where $\alpha$ is a quasi-isomorphism and $\tilde{\Phi}$ an isomorphism. The construction of such a factorization (see 6.3) is quite general and is useful in other situations (see e.g. [Wa], $[\mathrm{So}],[\mathrm{Ke}])$.

1.4. Here is a sheaf-theoretic interpretation due to A. Beilinson (private communication). He interprets $T_{1,2, \ldots, n}$ as $H^{1}(D, P)$, where $D$ is the filled $n$-gon and $P$ is the following subsheaf of the constant sheaf $V_{D}$ with fibre $V$. Cyclically label the vertices of $D$ by $\mathbb{Z} / n \mathbb{Z}$ and give $D$ the corresponding orientation. Let $U$ be the interior of $D$. Then $P$ has fibre $V$ on $U, l_{i}$ on the edge $(i, i+1)$, and $l_{i-1} \cap l_{i}$ at the vertex $i$.

Write $j_{U}: U \rightarrow D$ for the inclusion. Let $F_{U}$ be the constant sheaf on $U$ with fibre $F$. The symplectic form on $V$ induces a map $P \otimes P \rightarrow j_{U, !} F_{U}$, and thereby

$$
\cup: \operatorname{Sym}^{2} H^{1}(D, P) \rightarrow H^{2}\left(D, j_{U, !} F_{U}\right)=F .
$$

In section 8 , which can mostly be read independently from the rest of the work, we show that (4) is non-degenerate and in fact is minus our original bilinear form.

1.4.1. From this perspective, dihedral symmetry (1) is already clear, since the cyclic symmetry is manifest, while reversing the order of the Lagrangians reverses the orientation of $D$ and so changes the identification $H^{2}\left(D, j_{!} F_{U}\right)=F$ by a sign.

1.4.2. In 8.3 we explain how the chain condition (2) may be proved via a "bordism" between three polygons, in analogy to the proof of the additivity of the index of manifolds.

1.5. In the electronic version www.arxiv.org/math.SG/0505561/ of this paper, we explain how our quadratic form occurs naturally in the context of the Weil representation, which originally motivated this work. The relationship between the Maslov index and the Weil representation is well known (see $[\mathrm{LV}],[\mathrm{Li}],[\mathrm{Pe}],[\mathrm{Ra}],[\mathrm{Sou}], \ldots$ ), with $n=3$ Lagrangians being the key case. Our definitions allow a direct approach for any $n$.

1.6. I am grateful to V. Drinfeld for suggesting this subject, and to him and D. Arinkin for much useful advice. I also thank A. Beilinson for explaining his sheaf-theoretic reformulation, and M. Kamgarpour and B. Wieland for many interesting discussions.

\section{The Quadratic Space.}

2.1. Preliminaries. Our Lagragians $l_{1}, \ldots, l_{n}$ are indexed by $\mathbb{Z} / n \mathbb{Z}$. Think of $\mathbb{Z} / n \mathbb{Z}$ as the vertices of a graph whose set $\mathbb{E}$ of edges consists of pairs of consecutive numbers $\{i, i+1\}, i \in \mathbb{Z} / n \mathbb{Z}$. So the graph looks like an $n$-sided polygon.

An element

$$
v=\left(v_{\{i, i+1\}}\right) \in \bigoplus_{\{i, i+1\} \in \mathbb{E}} V
$$


may be thought of as a function $a: \mathbb{E} \rightarrow V$. We can form the 'derivative'

$$
\partial v=\left(\partial v_{i}\right) \in \bigoplus_{i \in \mathbb{Z} / n \mathbb{Z}} V \quad \partial v_{i}=v_{\{i, i+1\}}-v_{\{i-1, i\}} .
$$

Conversely, given $w=\left(w_{i}\right) \in \bigoplus_{i \in \mathbb{Z} / n \mathbb{Z}} V$, there is the obvious notion of an 'antiderivative' $\hat{w}$

$$
\hat{w}=\left(\hat{w}_{\{i, i+1\}}\right) \in \bigoplus_{\{i, i+1\} \in \mathbb{E}} V \quad \text { such that } \partial(\hat{w})=w .
$$

An anti-derivative exists so long as $\sum_{i \in \mathbb{Z} / n \mathbb{Z}} w_{i}=0$ in which case $\hat{w}$ is unique up to adding a constant function.

\subsection{Definitions.}

2.2.1. Definition. Let $K_{1,2, \ldots, n}$ be the kernel of the natural summation

$$
K_{1,2, \ldots, n}=\operatorname{ker}\left[\bigoplus_{i \in \mathbb{Z} / n \mathbb{Z}} l_{i} \stackrel{\Sigma}{\longrightarrow} V\right] .
$$

Any $w=\left(w_{i}\right) \in K_{1,2, \ldots, n}$ has an anti-derivative $\hat{w} \in \bigoplus_{\{i, i+1\} \in \mathbb{E}} V$, as in 2.1.

2.2.2. Definition. Define a bilinear form $q_{1,2, \ldots, n}$ on $K_{1,2, \ldots, n}$ by the formula

$$
q_{1,2, \ldots, n}(v, w)=\sum_{i \in \mathbb{Z} / n \mathbb{Z}} B\left(v_{i}, \hat{w}_{\{i, i+1\}}\right)
$$

for any choice of anti-derivative $\hat{w}$ (it is simple to check that the right-hand side of (8) is independent of this choice.)

Remark 1 . The bilinear form may equivalently be defined by

$$
q_{1,2, \ldots, n}(v, w)=\sum_{i \in \mathbb{Z} / n \mathbb{Z}} B\left(v_{i}, \hat{w}_{\{i-1, i\}}\right) .
$$

Indeed, the difference is

$$
\sum_{i \in \mathbb{Z} / n \mathbb{Z}} B\left(v_{i}, \hat{w}_{\{i, i+1\}}-\hat{w}_{\{i-1, i\}}\right)=\sum_{i \in \mathbb{Z} / n \mathbb{Z}} B\left(v_{i}, w_{i}\right),
$$

and $B\left(v_{i}, w_{i}\right)=0$ since $v_{i}, w_{i}$ lie in the same Lagrangian $l_{i}$.

Remark 2. In the definition (8) of $q_{1,2, \ldots, n}$, one may concretely choose $\hat{w}_{\{i, i+1\}}=$ $\sum_{j=1}^{i} w_{j}$, in which case the definition takes the simple form

$$
q_{1,2, \ldots, n}(v, w)=\sum_{i \geq j \geq 1}^{n} B\left(v_{i}, w_{j}\right)=\sum_{i>j>1}^{n} B\left(v_{i}, w_{j}\right) .
$$

We often use this version for calculations, but the natural symmetries of $q_{1,2, \ldots, n}$ are obscured.

Proposition 2. The bilinear form $q_{1,2, \ldots, n}$ is symmetric. 
Proof. "Summation by parts." Explicitly,

$$
\begin{aligned}
q_{1,2, \ldots, n}(v, w) & =\sum_{i \in \mathbb{Z} / n \mathbb{Z}} B\left(v_{i}, \hat{w}_{\{i, i+1\}}\right) \\
& =\sum_{i \in \mathbb{Z} / n \mathbb{Z}} B\left(\hat{v}_{\{i, i+1\}}-\hat{v}_{\{i-1, i\}}, \hat{w}_{\{i, i+1\}}\right) \\
& =\sum_{i \in \mathbb{Z} / n \mathbb{Z}} B\left(\hat{w}_{\{i, i+1\}}-\hat{w}_{\{i-1, i\}}, \hat{v}_{\{i-1, i\}}\right) \\
& =\sum_{i \in \mathbb{Z} / n \mathbb{Z}} B\left(w_{i}, \hat{v}_{\{i-1, i\}}\right)=q_{1,2, \ldots, n}(w, v) .
\end{aligned}
$$

The last equality is (9).

\subsubsection{Definition of the Quadratic Space $\left(T_{1,2, \ldots, n}, q_{1,2, \ldots, n}\right)$.}

$$
T_{1,2, \ldots, n}=K_{1,2, \ldots, n} / \operatorname{ker} q_{1,2, \ldots, n} .
$$

The induced non-degenerate bilinear form on $T_{1,2, \ldots, n}$ will still be called $q_{1,2, \ldots, n}$.

Let us give an explicit description of $T_{1,2, \ldots, n}$. The derivative (5) restricts to give a map

$$
\partial: \bigoplus_{\{i, i+1\} \in \mathbb{E}} l_{i} \cap l_{i+1} \rightarrow K_{1,2, \ldots, n} .
$$

It is clear from the definition (8) that $\operatorname{ker} q_{1,2, \ldots, n} \supset \operatorname{im} \partial$.

Proposition 3. We have $\operatorname{ker} q_{1,2, \ldots, n}=\operatorname{im} \partial$. In other words, $T_{1,2, \ldots, n}$ is the cohomology $H^{0}(C)$ at the center term of the complex

$$
C=\left[\bigoplus_{\{i, i+1\} \in \mathbb{E}} l_{i} \cap l_{i+1} \stackrel{\partial}{\longrightarrow} \bigoplus_{i \in \mathbb{Z} / n \mathbb{Z}} l_{i} \stackrel{\Sigma}{\longrightarrow} V\right]
$$

which we consider to lie in degrees $-1,0,1$.

The proof will be given in section 3 , where we also show that $q_{1,2, \ldots, n}$ is induced by a quasi-isomorphism between $C$ and $C^{*}$, and give a formula for the dual form on $T_{1,2, \ldots, n}^{*}$.

\section{Corollary.}

$$
\operatorname{dim} T_{1,2, \ldots, n}=(n-2) \frac{\operatorname{dim} V}{2}-\sum_{i \in \mathbb{Z} / n \mathbb{Z}} \operatorname{dim} l_{i} \cap l_{i+1}+2 \operatorname{dim} \bigcap_{i \in \mathbb{Z} / n \mathbb{Z}} l_{i} .
$$

2.2.4. Definition. The symbol $\tau\left(l_{1}, l_{2}, \ldots, l_{n}\right)$ denotes the class of the quadratic space $\left(T_{1,2, \ldots, n}, q_{1,2, \ldots, n}\right)$ in $W(F)$, called the Maslov index of $l_{1}, \ldots, l_{n}$.

In section 7 we will verify that this Maslov index equals Kashiwara's. 


\section{Proof of Proposition 3; \\ The Dual Form; Homotopy Equivalence of $C$ and $C^{*}$.}

In this section we give an algebraic proof of Proposition 3 (a sheaf-theoretic argument is given in section 8). The proof allows us to write down a formula for the dual form on $T_{1,2, \ldots, n}^{*}$ in 3.2. It also implies that the complexes $C$ and $C^{*}$ are isomorphic in the derived category of complexes of vector spaces, this isomorphism inducing $q_{1,2, \ldots, n}$ on $H^{0}(C)$. In 3.3 we give an explicit, though non-canonical, symmetric quasiisomorphism $C \rightarrow C^{*}$.

3.1. Proof of Proposition 3. Let $\Phi$ be the linear map $\Phi: H^{0}(C) \rightarrow H^{0}\left(C^{*}\right)=H^{0}(C)^{*}$ such that

$$
q_{1,2, \ldots, n}(v, w)=\langle v, \Phi w\rangle .
$$

We want to show that $\Phi$ is an isomorphism.

The situation is expressed by the following commutative diagram, in which the top row is $C$ and the bottom row $C^{*}$; every row is a complex.

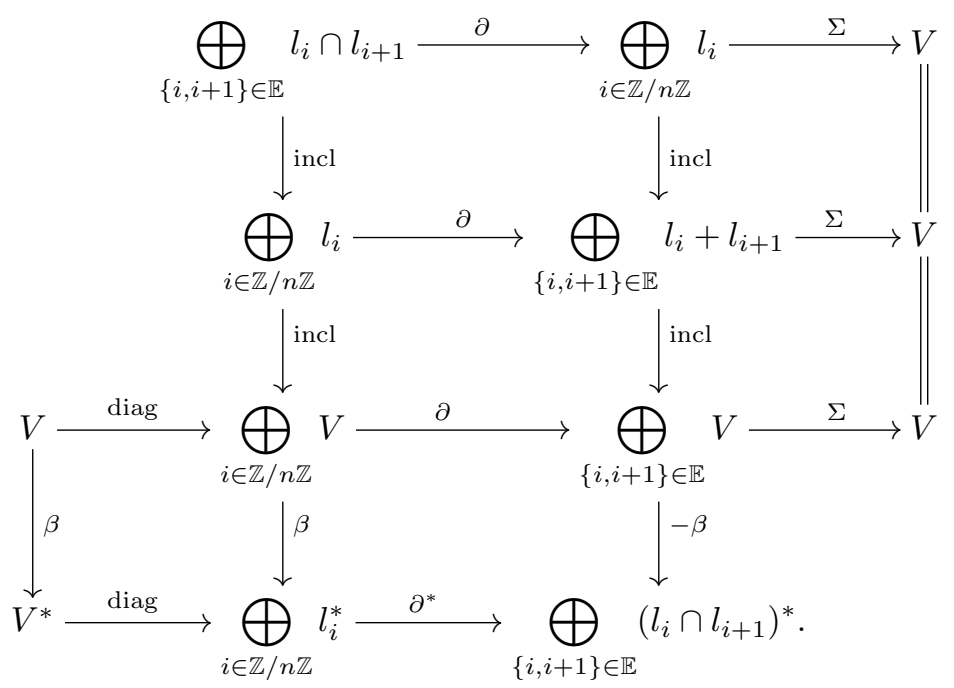

Here "incl" means summand-by-summand inclusion and $\beta: x \mapsto B(-, x)$.

By definition (8) of $q_{1,2, \ldots, n}, \Phi: H^{0}(C) \rightarrow H^{0}\left(C^{*}\right)$ factors as

$$
\Phi=\beta \circ \partial^{-1} \circ \text { incl } \circ \text { incl. }
$$

Denote by $W$ the cohomology at the center term of the second row. The first two rows of the diagram constitute a quasi-isomorphism of complexes, because the map "incl" between them is injective with acyclic cokernel. In particular we obtain an isomorphism incl: $H^{0}(C) \rightarrow W$.

On the other hand, the last three rows of the diagram form a short exact sequence of complexes; since the third row is exact, the boundary map $\delta=\mathrm{incl}^{-1} \circ \partial \circ$ $\beta^{-1}: H^{0}\left(C^{*}\right) \rightarrow W$ is an isomorphism.

Thus $\Phi$ factors through isomorphisms

$$
\Phi: H^{0}(C) \stackrel{\text { incl }}{\longrightarrow} W \stackrel{\delta^{-1}}{\longrightarrow} H^{0}\left(C^{*}\right) .
$$


3.2. The Dual Form. Write $q_{1,2, \ldots, n}^{*}$ for the quadratic form on $T_{1,2, \ldots, n}^{*}$, induced, in the notation of 3.1, by the isomorphism $\Phi^{-1}: H^{0}\left(C^{*}\right) \rightarrow H^{0}(C)$. Let us record a formula for $q_{1,2, \ldots, n}^{*}$ which follows from the factorization (16).

We will not need it for the rest of this article.

3.2.1. Define $S:=\left\{x \in \bigoplus_{i \in \mathbb{Z} / n \mathbb{Z}} V \mid x_{i+1}-x_{i} \in l_{i}+l_{i+1}\right\} . T_{1,2, \ldots, n}^{*}$ is a quotient of $S$ : the map $S \rightarrow T_{1,2, \ldots, n}^{*}$ is given by the last two rows of (15), in which $S=\operatorname{ker}(-\beta \circ \partial)$ and $T_{1,2, \ldots, n}^{*}=\operatorname{ker}\left(\partial^{*}\right) / \mathrm{im}(\operatorname{diag})$. We will describe $q_{1,2, \ldots, n}^{*}$ pulled back to $S$.

3.2.2. Suppose first given $x_{i}, x_{i+1} \in V$ with $x_{i+1}-x_{i} \in l_{i}+l_{i+1}$. Define a functional $\varepsilon\left(x_{i}, x_{i+1}\right)$ on $l_{i}+l_{i+1}$ as follows. For $v \in l_{i}+l_{i+1}$, write $v=a+b$, with $a \in l_{i}$, $b \in l_{i+1}$. Then

$$
\left\langle\varepsilon\left(x_{i}, x_{i+1}\right), v\right\rangle:=B\left(a, x_{i}\right)+B\left(b, x_{i+1}\right) .
$$

It is easy to verify that this quantity is independent of the choice of $a, b$.

Proposition 4. As a bilinear form on $S$,

$$
q_{1,2, \ldots, n}^{*}(x, y)=\sum_{i \in \mathbb{Z} / n \mathbb{Z}}\left\langle\varepsilon\left(x_{i}, x_{i+1}\right), y_{i+1}-y_{i}\right\rangle .
$$

3.3. An Explicit Quasi-Isomorphism. Here is one particular quasi-isomorphism $\Phi: C \rightarrow C^{*}$ inducing $q_{1,2, \ldots, n}$ on $H^{0}(C)$ :

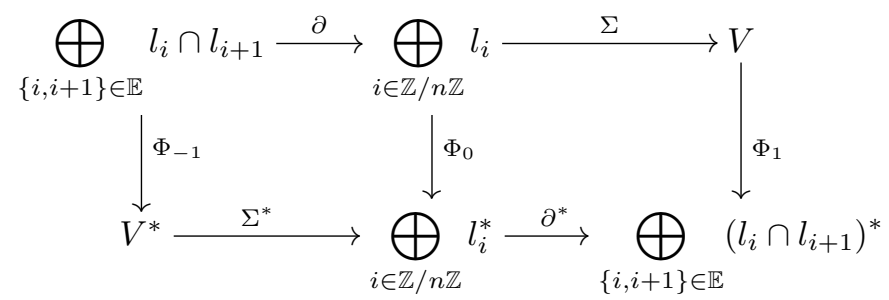

where

$$
\begin{aligned}
\left\langle\Phi_{-1}(a), v\right\rangle & =\left\langle a, \Phi_{1}(v)\right\rangle=B\left(a_{\{n, 1\}}, v\right) \\
\left\langle\Phi_{0}(a), b\right\rangle & =\left\langle a, \Phi_{0}(b)\right\rangle=\frac{1}{2} \sum_{i \geq j \geq 1}^{n}\left(B\left(a_{i}, b_{j}\right)+B\left(b_{i}, a_{j}\right)\right) .
\end{aligned}
$$

The fact that $\Phi$ induces $q_{1,2, \ldots, n}$ follows from formula (10).

Remark. $\Phi=\Phi^{*}$. The isomorphism

$$
H^{-1}(C) \oplus H^{0}(C) \oplus H^{1}(C) \longrightarrow H^{-1}(C)^{*} \oplus H^{0}(C)^{*} \oplus H^{1}(C)^{*}
$$

implicit in diagram (15) is symmetric, so any $\Phi: C \rightarrow C^{*}$ inducing it may be symmetrized by $\Phi \mapsto \frac{1}{2}\left(\Phi+\Phi^{*}\right)$. 


\section{Dihedral Symmetry (1).}

The space $K_{1,2, \ldots, n}$ can be canonically identified with $K_{2,3, \ldots, n, 1}$ and $K_{n, n-1, \ldots, 1}$, as is obvious from definition (7).

Proposition 5. Under these identifications, $q_{2,3, \ldots, n, 1}=q_{1,2, \ldots, n}=-q_{n, n-1, \ldots, 1}$.

Proof. The first equality (cyclic symmetry) is obvious from the definition (8) of $q_{1,2, \ldots, n}$. Reversing the order of the Lagrangians is equivalent to replacing $\partial$ by $-\partial$, and therefore $\hat{w}$ by $-\hat{w}$ in equation (8).

\section{The Chain Condition (2).}

Proposition 6. Fix $k \in\{2, \ldots, n\}$.

1. If $l_{1} \cap l_{k}=0$ then $T_{1,2, \ldots, k} \oplus T_{1, k, \ldots, n} \cong T_{1,2, \ldots, n}$ isometrically.

2. Without conditions, $T_{1,2, \ldots, k} \oplus T_{1, k, \ldots, n}$ is a quadratic subquotient of $T_{1,2, \ldots, n}$. Therefore, by Lemma 1, $\tau\left(l_{1}, l_{2}, \ldots, l_{k}\right)+\tau\left(l_{1}, l_{k}, \ldots, l_{n}\right)=\tau\left(l_{1}, l_{2}, \ldots, l_{n}\right)$.

Proof. The equivalence is induced by the natural map

$$
\bigoplus_{i \in\{1,2, \ldots, k\}} l_{i} \oplus \bigoplus_{i \in\{1, k, \ldots, n\}} l_{i} \stackrel{s}{\longrightarrow} \bigoplus_{i \in\{1,2, \ldots, n\}} l_{i}
$$

that is the identity on each summand. More precisely, consider the map of short exact sequences

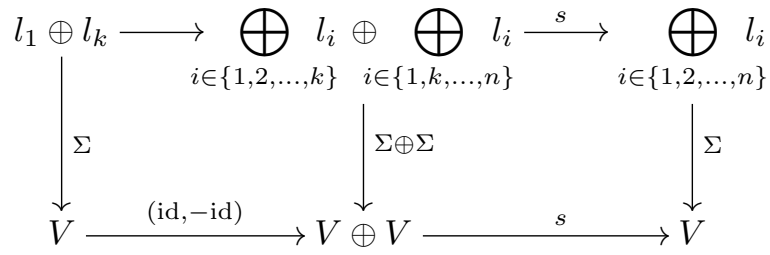

The snake lemma gives an exact sequence

$$
l_{1} \cap l_{k} \stackrel{(r,-r)}{\longrightarrow} K_{1,2, \ldots, k} \oplus K_{1, k, \ldots, n} \stackrel{s}{\longrightarrow} K_{1,2, \ldots, n} \stackrel{\delta}{\longrightarrow} V /\left(l_{1}+l_{k}\right) .
$$

Here $r=(-\mathrm{id}, \mathrm{id}): l_{1} \cap l_{k} \rightarrow l_{1} \oplus l_{k}$ and the boundary $\delta$ is given by

$$
\delta(v)=\sum_{i=1}^{k} v_{i} \bmod \left(l_{1}+l_{k}\right)
$$

Lemma 7. The map $s: K_{1,2, \ldots, k} \oplus K_{1, k, \ldots, n} \rightarrow K_{1,2, \ldots, n}$ is an isometry.

Proof. One sees immediately from the explicit formula (10) that $s$ restricted to each summand is an isometry, and that for any $(v, w) \in K_{1,2, \ldots, k} \oplus K_{1, k, \ldots, n}$ we have $q_{1,2, \ldots, n}(s(v, 0), s(0, w))=0$. No more is required.

Transverse Case. If $l_{1} \cap l_{k}=0$ then, by the exactness of (19), $s$ is an isometric isomorphism. Passing to the non-degenerate quotients establishes part 1 of the proposition.

General Case. Consider the map $r: l_{1} \cap l_{k} \rightarrow K_{1,2, \ldots, k}$ as in (19). By Proposition 3, the image lies in ker $q_{1,2, \ldots, k}$, so, since $s$ is an isometry, im $s \circ r$ is isotropic in $K_{1,2, \ldots, n}$ and $\operatorname{im} s \subset(\operatorname{im} s \circ r)^{\perp}$.

Lemma 8. The image of $s$ is exactly $(\operatorname{im} s \circ r)^{\perp}$. 
Proof. According to formula (10), if $v \in l_{1} \cap l_{k}$ and $w \in K_{1,2, \ldots, n}$, then

$$
q_{1,2, \ldots, n}(s \circ r(v), w)=\sum_{j=1}^{k} B\left(v, w_{j}\right) .
$$

This quantity vanishes for all $v \in l_{1} \cap l_{k}$ if and only if $\sum_{i=1}^{k} w_{k}$ lies in $l_{1}+l_{k}$; according to (20) this just means $\delta(w)=0$ or equivalently $w \in \operatorname{im} s$.

Let $I$ denote the image of im $s \circ r$ in $T_{1,2, \ldots, n}$. We have constructed a surjective isometry

and therefore

$$
s: K_{1,2, \ldots, k} \oplus K_{1, k, \ldots, n} \longrightarrow I^{\perp} / I
$$

$$
s: T_{1,2, \ldots, k} \oplus T_{1, k, \ldots, n} \stackrel{\cong}{\longrightarrow} I^{\perp} / I .
$$

\section{Local Constancy (3).}

In this section, the ground field $F$ is a local field.

6.1. In order to prove (3) we will use the following well known fact:

Lemma 9. Let $Q(X)$ be the space of non-degenerate symmetric bilinear forms on a fixed vector space $X$. Then the natural $G L(X)$ action on $Q(X)$ has open orbits. In other words, in a continuous family of quadratic spaces of fixed rank, the isomorphism class is locally constant.

Proof. It suffices to check that for any $q \in Q(X)$ the map $\lambda: G L(X) \rightarrow Q(X)$ given by $\lambda(g)(x, x)=q(g x, g x)$ has surjective differential at $1 \in G L(X)$. The differential $\lambda_{*}: \mathfrak{g l}(X) \rightarrow \operatorname{Sym}^{2}\left(X^{*}\right)$ is given by $\lambda_{*}(A)(x, x)=2 q(A x, x)$; it is surjective since $q$ is non-degenerate.

We define in this section another quadratic space $\tilde{T}_{1,2, \ldots, n}$ whose class in $W(F)$ is the same as that of $T_{1,2, \ldots, n}$, but whose dimension is locally constant in $l_{1}, \ldots, l_{n}$ when the dimensions $\operatorname{dim} l_{i} \cap l_{i+1}$ are fixed. Then property (3) follows from Lemma 9. Note that, by (14), the dimension of $T_{1,2, \ldots, n}$ itself varies with $\operatorname{dim}\left(l_{1} \cap \cdots \cap l_{n}\right)$.

Remark. One could use $\tilde{T}_{1,2, \ldots, n}$ to define the Maslov index, but then the dihedral symmetry (1) would not be immediately clear.

We will first give a concrete formula (22) for the quadratic form $\tilde{q}_{1,2, \ldots, n}$ on $\tilde{T}_{1,2, \ldots, n}$, but in section 6.3 we describe its origin in a general construction.

6.2. Definition. Define a symmetric bilinear form $\tilde{q}_{1,2, \ldots, n}$ on

$$
\tilde{K}_{1,2, \ldots, n}=V^{*} \oplus \bigoplus_{i \in \mathbb{Z} / n \mathbb{Z}} l_{i}
$$

by

$$
\tilde{q}_{1,2, \ldots, n}(v \oplus a, v \oplus a)=2 \sum_{i \in \mathbb{Z} / n \mathbb{Z}}\left\langle v, a_{i}\right\rangle+\sum_{i \geq j \geq 1} B\left(a_{i}, a_{j}\right) .
$$


Define

$$
\tilde{T}_{1,2, \ldots, n}=\tilde{K}_{1,2, \ldots, n} / \operatorname{ker} \tilde{q}_{1,2, \ldots, n}
$$

and again write $\tilde{q}_{1,2, \ldots, n}$ for the non-degenerate form on $\tilde{T}_{1,2, \ldots, n}$.

To describe $\tilde{T}_{1,2, \ldots, n}$ explicitly, let

$$
\tilde{\partial}=\left(\Phi_{-1}, \partial\right): \bigoplus_{\{i, i+1\} \in \mathbb{E}} l_{i} \cap l_{i+1} \rightarrow \tilde{K}_{1,2, \ldots, n} .
$$

Here $\Phi_{-1}: \bigoplus\left(l_{i} \cap l_{i+1}\right) \rightarrow V^{*}$ as in $(17,18)$ and $\partial: \bigoplus\left(l_{i} \cap l_{i+1}\right) \rightarrow \bigoplus l_{i}$ as in (12).

Proposition 10. With notation as above:

1. We have $\operatorname{ker} \tilde{q}_{1,2, \ldots, n}=\operatorname{im} \tilde{\partial}$, and $\tilde{\partial}$ is injective. Therefore

$$
\operatorname{dim} \tilde{T}_{1,2, \ldots, n}=(n+2) \frac{\operatorname{dim} V}{2}-\sum_{\{i, i+1\} \in \mathbb{E}} \operatorname{dim} l_{i} \cap l_{i+1} .
$$

2. $T_{1,2, \ldots, n}$ is a quadratic subquotient of $\tilde{T}_{1,2, \ldots, n}$ by the image of $V^{*} \subset \tilde{K}_{1,2, \ldots, n}$. Therefore, by Lemma $1, T_{1,2, \ldots, n}$ and $\tilde{T}_{1,2, \ldots, n}$ have the same class in $W(F)$.

Proof of Proposition 10. It is simple to check Proposition 10 directly, making reference to the quasi-isomorphism $(17,18)$; in the notation there,

$$
\tilde{q}_{1,2, \ldots, n}(v \oplus a, w \oplus b)=\langle v, \Sigma b\rangle+\left\langle a, \Phi_{0} b\right\rangle+\left\langle a, \Sigma^{*} w\right\rangle .
$$

Otherwise, one can feed that quasi-isomorphism into the following general construction.

6.3. Let $C$ be a complex of finite-dimensional vector spaces. Suppose given a symmetric quasi-isomorphism $\Phi: C \rightarrow C^{*}$. Then we construct another such complex $D$ and a commutative diagram

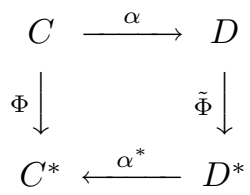

where $\alpha$ is a quasi-isomorphism and $\tilde{\Phi}$ a symmetric isomorphism.

Remark. Instead of vector spaces, one can consider projective modules over an associative ring with anti-involution. The construction still works, if one everywhere replaces "quasi-isomorphism" with "homotopy equivalence" and "acyclic" with "homotopy equivalent to zero."

In fact, [Wa, Theorem 9.4] generalizes this construction to complexes over any "exact category with duality containing $\frac{1}{2}$."

In the context of complexes of locally free modules over a commutative ring, an alternative local construction of a diagram similar to (24) can be found in [So], Corollaire 2.2. See also $[\mathrm{Ke}]$. 
Construction. The cone of $\Phi$ is an acyclic complex including

$\cdots C^{-1} \oplus\left(C^{2}\right)^{*} \stackrel{f_{-1}}{\longrightarrow} C^{0} \oplus\left(C^{1}\right)^{*} \stackrel{f_{0}}{\longrightarrow}\left(C^{0}\right)^{*} \oplus C^{1} \stackrel{f_{1}}{\longrightarrow}\left(C^{-1}\right)^{*} \oplus C^{2} \ldots$

where

$$
f_{-1}=f_{1}^{*}=\left(\begin{array}{cc}
d & 0 \\
-\Phi & d^{*}
\end{array}\right) \quad f_{0}=\left(\begin{array}{cc}
\Phi & d^{*} \\
d & 0
\end{array}\right) .
$$

Define

$$
D^{0}=\left(C^{0} \oplus\left(C^{1}\right)^{*}\right) / \operatorname{im} f_{-1} .
$$

Then $f_{0}$ gives a symmetric isomorphism $f_{0}: D^{0} \rightarrow\left(D^{0}\right)^{*}$.

Moreover, we get a chain of complexes

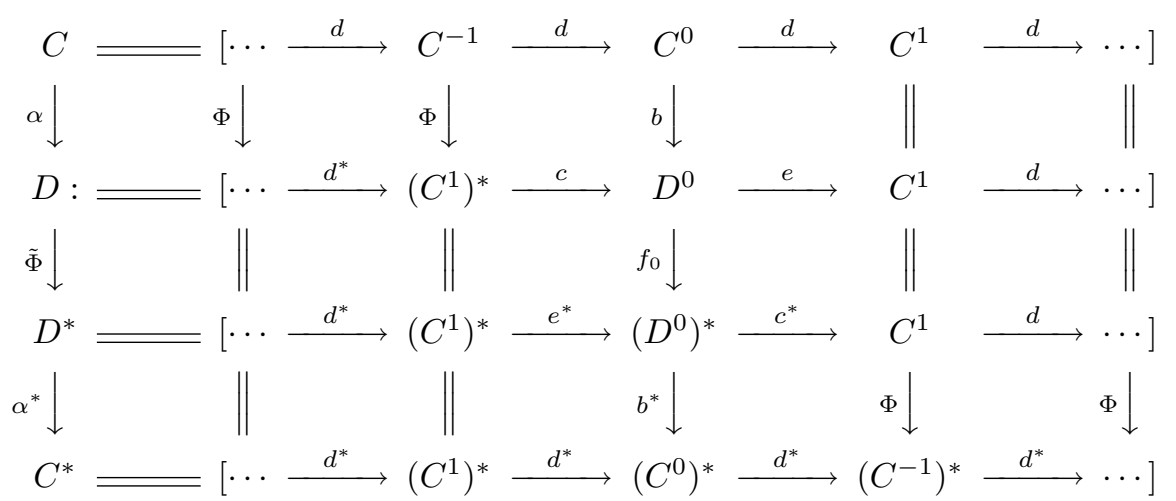

satisfying our requirements. The maps $b: C^{0} \rightarrow D^{0}$ and $c:\left(C^{1}\right)^{*} \rightarrow D^{0}$ are the natural ones from the definition of $D^{0}$, while $e: D^{0} \rightarrow C^{1}$ is induced by $(d, 0): C^{0} \oplus$ $\left(C^{1}\right)^{*} \rightarrow C^{1}$.

6.4. In our particular case, $f_{-1}=\tilde{\partial}$ is injective; we obtain a complex $D$ with $D^{0}=$ $\tilde{K}_{1,2, \ldots, n} /$ im $\tilde{\partial}$ and a symmetric isomorphism $f_{0}: D^{0} \rightarrow\left(D^{0}\right)^{*}$ inducing the form $\tilde{q}_{1,2, \ldots, n}$. Moreover, $H^{0}(D)=H^{0}(C)=T_{1,2, \ldots, n}$ is the quadratic subquotient of $D^{0}=$ $\tilde{T}_{1,2, \ldots, n}$ by the image of $c$ in diagram (26). This completes the proof of Proposition 10 .

\section{Relation to Kashiwara's Construction.}

Let us recall the construction of Kashiwara's Maslov index, which we denote by $\tau^{\text {Kash }}\left(l_{1}, \ldots, l_{n}\right)$. First, for $n=3, \tau^{\text {Kash }}\left(l_{1}, l_{2}, l_{3}\right) \in W(F)$ is represented by the symmetric bilinear form $q_{1,2,3}^{\text {Kash }}$ on $T_{1,2,3}^{\text {Kash }}=l_{1} \oplus l_{2} \oplus l_{3}$ with

$$
q_{1,2,3}^{\mathrm{Kash}}(v, w)=\frac{1}{2}\left(B\left(v_{1}, w_{2}-w_{3}\right)+B\left(v_{2}, w_{3}-w_{1}\right)+B\left(v_{3}, w_{1}-w_{2}\right)\right) .
$$

The definition can then be extended inductively to any $n$ by the chain condition

$$
\tau^{\mathrm{Kash}}\left(l_{1}, l_{2}, \ldots, l_{n}\right):=\tau^{\mathrm{Kash}}\left(l_{1}, l_{2}, \ldots, l_{k}\right)+\tau^{\mathrm{Kash}}\left(l_{1}, l_{k}, \ldots, l_{n}\right)
$$

for any $k \in\{3, \ldots, n-1\}$.

Proposition 11. $\tau\left(l_{1}, l_{2}, \ldots, l_{n}\right)=\tau^{\mathrm{Kash}}\left(l_{1}, l_{2}, \ldots, l_{n}\right)$.

Proof. Using the chain properties (2),(27), we need only consider $n=3$. We show that $T_{1,2,3}$ is a quadratic subquotient of $T_{1,2,3}^{\mathrm{Kash}}$. 
Lemma 12. $I=l_{1} \subset T_{1,2,3}^{\mathrm{Kash}}$ is an isotropic subspace;

$$
I^{\perp}=\left\{\left(v_{1}, v_{2}, v_{3}\right) \text { with } v_{2}-v_{3} \in l_{1}\right\} .
$$

Lemma 13. The map

$$
\left(v_{1}, v_{2}, v_{3}\right) \mapsto\left(v_{2}-v_{3},-v_{2}, v_{3}\right)
$$

defines an isometric surjection $I^{\perp} \rightarrow K_{1,2,3}$.

The lemmas, which may be checked directly, combine to give an isometric isomorphism from the non-degenerate part of $I^{\perp} / I$ to $T_{1,2,3}$. Lemma 1 concludes the proof of Proposition 11.

\section{The Maslov Index via Sheaves.}

In this section we verify basic properties of Beilinson's construction (see 1.4).

Throughout we use the following notation: if $X$ is a topological space and $W$ a vector space then $W_{X}$ is the constant sheaf on $X$ with fibre $W$; if $Y$ is a subset of $X$ let $j_{Y}$ denote its inclusion. $\mathbb{D}$ is the Verdier dualizing operator.

8.1. Non-Degeneracy. Since $\left(j_{U, !} F_{U}\right)[2]$ is the dualizing complex on $D$, the map $P \otimes P \rightarrow j_{U, !} F_{U}$ induced by the symplectic form on $V$ defines a map $\phi: P \rightarrow(\mathbb{D} P)[-2]$. The fact that the bilinear form (4) on $H^{1}(D, P)$ is non-degenerate follows from the following lemma.

Lemma 14. The map $\phi: P \rightarrow(\mathbb{D} P)[-2]$ is an isomorphism.

Proof. The question is local; it suffices to check that $\phi$ is an isomorphism over the open sets of the form

$$
U_{i}:=U \cup(i-1, i) \cup\{i\} \cup(i, i+1) \quad i \in \mathbb{Z} / n \mathbb{Z}
$$

since these cover $D$. Write $M:=l_{i-1} \cap l_{i}$ and choose a decomposition

$$
V=M \oplus L \oplus L^{\prime} \oplus M^{\prime}
$$

such that $l_{i-1}=M \oplus L$ and $l_{i}=M \oplus L^{\prime}$. Write $a, b$ for the inclusions

$$
U \stackrel{a}{\longrightarrow} U \cup(i-1, i) \stackrel{b}{\longrightarrow} U_{i} .
$$

Then on $U_{i}$ we have

$$
P=b_{*} a_{*} M_{U} \oplus b_{!} a_{*} L_{U} \oplus b_{*} a_{!} L_{U}^{\prime} \oplus b_{!} a_{!} M_{U}^{\prime} .
$$

To compute $\mathbb{D} P$, note, for example, that $\mathbb{D} b_{*} a_{*} M_{U}=b_{!} a_{!} \mathbb{D} M_{U}=b_{!} a_{!}\left(M_{U}^{*}[2]\right)$. Therefore, dualizing (28) and reversing the order of the summands, we obtain

$$
(\mathbb{D} P)[-2]=b_{*} a_{*} M^{\prime *} \oplus b_{!} a_{*} L_{U}^{\prime *} \oplus b_{*} a_{!} L_{U}^{*} \oplus b_{!} a_{!} M_{U}^{*} .
$$

We observe that $\phi$ is an upper-triangular matrix with respect to the decompositions (28), (29); moreover, the diagonal entries are isomorphisms, since the symplectic form gives isomorphisms $L^{\prime} \cong L^{*}$ and $M^{\prime} \cong M^{*}$. 


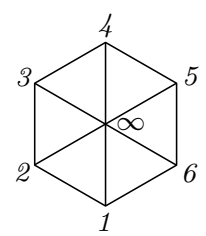

FiguRE 1. Triangulation of $D$ for $n=6$ Lagrangians.

8.2. Equivalence with Algebraic Definition 2.2.3. The cellular cochain complex arising from the sheaf $P$ is evidently our original complex $C$ in (13). Therefore $H^{1}(D, P)=T_{1,2, \ldots, n}$.

As for the bilinear form, let us calculate explicitly the cup product (4). We triangulate $D$ by introducing a central vertex $\infty$ and drawing radii, as in Figure 1.

Corresponding to this triangulation, a 1-cochain $\alpha$ with values in $P$ consists of choices of $\alpha_{\{i, i+1\}} \in l_{i}$ and $\alpha_{\{i, \infty\}} \in V$. The cocycle condition amounts to

$$
\alpha_{\{i+1, \infty\}}-\alpha_{\{i, \infty\}}=-\alpha_{\{i, i+1\}} .
$$

A cocycle corresponds to an element $v \in T_{1,2, \ldots, n}$ by $v_{i}=\alpha_{\{i, i+1\}}$, so the cocycle condition implies $\alpha_{\{i+1, \infty\}}=-\hat{v}_{\{i, i+1\}}$ in the notation of (6).

A 2-cocycle $\gamma$ with values in $j_{U, !} F_{U}$ consists of choices of $\gamma_{\{i, i+1, \infty\}} \in V$. The evaluation $H^{2}\left(D, j_{!} F_{U}\right) \rightarrow F$ is given by $\gamma \mapsto \sum_{i \in \mathbb{Z} / n \mathbb{Z}} \gamma_{\{i, i+1, \infty\}}$. Given 1-cocycles $\alpha, \beta$ the 2-cocycle $\gamma=\alpha \cup \beta$ is given by the usual formula

$$
\gamma_{\{i, i+1, \infty\}}=B\left(\alpha_{\{i, i+1\}}, \beta_{\{i+1, \infty\}}\right) .
$$

All together, the pairing (4) is given on cocycles by

$$
(\alpha, \beta) \mapsto \sum_{i \in \mathbb{Z} / n \mathbb{Z}} B\left(\alpha_{\{i, i+1\}}, \beta_{\{i+1, \infty\}}\right)=\sum_{i \in \mathbb{Z} / n \mathbb{Z}} B\left(v_{i},-\hat{w}_{\{i, i+1\}}\right)=-q_{1,2, \ldots, n}(v, w)
$$

where $v, w \in T_{1,2, \ldots, n}$ are the classes of $\alpha, \beta$. The last equality is the definition (8) of $q_{1,2, \ldots, n}$.

Remark. The asymmetric formula (10) appears if we choose an asymmetric triangulation of $D$, namely, if we draw all the diagonals from the vertex 1 .

8.3. Chain Condition. Let us write $\left(D_{1}, P_{1}\right),\left(D_{2}, P_{2}\right),\left(D_{3}, P_{3}\right)$ for the polygons and sheaves corresponding to the collections $\left\{l_{1}, \ldots, l_{k}\right\},\left\{l_{1}, l_{k}, \ldots, l_{n}\right\}$, and $\left\{l_{1}, \ldots, l_{n}\right\}$.

Here is a proof of the chain condition (2) that is very similar to the proof of the additivity of the index of manifolds. Namely, we describe a "bordism" $(Y, \hat{P})$ from $\left(D_{1} \sqcup D_{2}, P_{1} \oplus P_{2}\right)$ to $\left(D_{3}, P_{3}\right)$, and argue that the image of $H^{1}(Y, \hat{P})$ in $H^{1}\left(D_{1}, P_{1}\right) \oplus$ $H^{1}\left(D_{2}, P_{2}\right) \oplus H^{1}\left(\bar{D}_{3}, P_{3}\right)$ is isotropic of half the total dimension; therefore the total quadratic space is hyperbolic. Here $\bar{D}_{3}$ is $D_{3}$ with opposite orientation.

In Figure 2 we show $Y$, with $n=4, k=3$ for concreteness. The sheaf $\hat{P}$ on $Y$ is constructible with respect to the pictured cell decomposition, with stalks as follows: $V$ on the interior of $Y$ and on the open top and bottom faces; $l_{1}, l_{2}, l_{3}, l_{4}$ on the open front, left, back, and right faces respectively. The stalk over any other cell is 


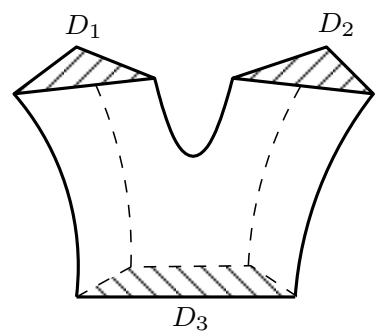

Figure 2. A "bordism" $Y$ between $D_{1} \sqcup D_{2}$ and $D_{3}$.

the intersection of the stalks over all adjacent cells, and all the restriction maps are inclusions.

Define $\hat{D}:=D_{1} \sqcup D_{2} \sqcup \bar{D}_{3} \subset Y$, and write $i$ for $j_{\hat{D}}$ and $j$ for $j_{Y-\hat{D}}$. Let $\hat{U}$ be the interior of $Y$. We orient $Y$ compatibly with the orientation of $\hat{D}$. The dualizing complex on $Y$ is then $j_{\hat{U}, !} F_{\hat{U}}[3]$. The symplectic form induces a pairing $\hat{P} \otimes j_{!} j ! \hat{P} \rightarrow$ $j_{\hat{U}, !} F_{\hat{U}}[3]$ and therefore a map

$$
\hat{\phi}: j ! j ! \hat{P} \rightarrow(\mathbb{D} \hat{P})[-3] .
$$

Lemma 15. $\hat{\phi}$ is an isomorphism. In particular, it induces isomorphisms

$$
H^{m}(Y, j ! j ! \hat{P}) \cong H^{3-m}(Y, \hat{P})^{*}
$$

for $m=0,1,2,3$.

Proof. This can be proved in the same way as Lemma 14. In fact, one can reduce to Lemma 14: the pair $(Y, \hat{P})$ is locally isomorphic to $\left(D \times I, P \otimes F_{I}\right)$, where $I$ is the the closed interval, and locally $\hat{\phi}$ is the isomorphism

$$
\hat{\phi}=\phi \otimes \mathrm{id}: P \otimes j_{I-\partial I, !} F_{I-\partial I} \rightarrow(\mathbb{D} P)[-2] \otimes\left(\mathbb{D} F_{I}\right)[-1]=\left(\mathbb{D}\left(P \otimes F_{I}\right)\right)[-3] .
$$

Proposition 16. The image of $\pi: H^{1}(Y, \hat{P}) \rightarrow H^{1}\left(\hat{D}, i^{*} \hat{P}\right)$ is isotropic of half the dimension of the total space.

Proof. It follows from the commutativity of the diagram

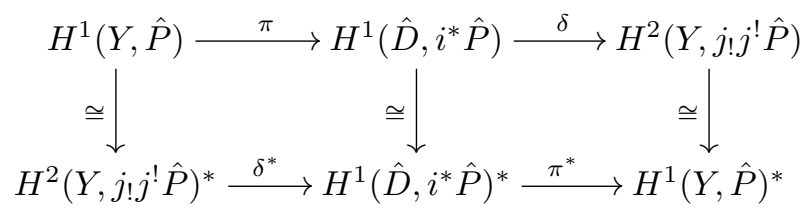

and the exactness of the rows. 


\section{References}

[CLM] S. Cappell, R. Lee, and E. Miller. "On the Maslov Index." Comm. Pure Appl. Math. 47 (1994), 121-186.

[KS] M. Kashiwara and P. Schapira. Sheaves on Manifolds. Berlin; New York: Springer, 1990.

[Ke] G. Kempf. "Deformations of Semi-Euler Characteristics." American J. of Math. 114 (1992), 973-978.

[Li] G. Lion. "Indice de Maslov et représentation de Weil." Trois textes sur les representations des groupes nilpotents et resolubles. Publications Mathématiques de l'Université Paris VII (1978).

[LV] G. Lion and M. Vergne, The Weil representation, Maslov index and Theta series. Progress in Math 6. Boston: Birkhaüser, 1980.

[Pe] P. Perrin. "Représentations de Schrödinger, indice de Maslov et groupe metaplectique." Non Commutative Harmonic Analysis and Lie Groups (Marseille-Luminy, 1980), 370-407. Lecture Notes in Math 880. Berlin; New York: Springer, 1981.

[Ra] R. Ranga Rao. "On some explicit formulas in the theory of Weil representations." Pacific J. Math. 157 (1993), 335-371.

[So] C. Sorger. "La semi-caractéristique d'Euler-Poincaré des faisceaux $\omega$-quadratiques sur un schéma de Cohen-Macaulay." Bull. Soc. Math. France 122 (1994), 225-233.

[Sou] J.-M. Souriau. "Construction explicite de l'indice de Maslov, applications." Group Theoretical Methods in Physics (Fourth International Colloquium, Nijmegen, 1975), 117-148. Lecture Notes in Physics 50. Berlin; New York: Springer, 1976.

[Wa] C. Walter. "Grothendieck-Witt groups of triangulated categories." Preprint, July 1, 2003, K-theory Preprint Archives, http://www.math.uiuc.edu/K-theory/0589/.

Merton College, Oxford University, Oxford OX1 4JD, UK

E-mail address: Joaquin. Thomas@aya.yale.edu 